\title{
Searching Ultra-compact Pulsar Binaries with Abnormal Timing Behavior
}

\author{
B. P. Gong ${ }^{1}$, Y. P. Li $^{2}$, J. P. Yuan ${ }^{3}$, J. Tian ${ }^{1,4}$, Y. Y. Zhang ${ }^{1}$, D. Li ${ }^{5,6}$, B. Jiang $^{7}$, X. D. Li ${ }^{7}$, H. G. Wang ${ }^{8}$, Y. C. Zou ${ }^{1}$, and L. J. Shao ${ }^{9}$ \\ ${ }^{1}$ Department of Physics, Huazhong University of Science and Technology, Wuhan 430074, People's Republic of China; bpgong@ mail.hust.edu.cn \\ ${ }^{2}$ Key Laboratory for Research in Galaxies and Cosmology, Shanghai Astronomical Observatory, Chinese Academy of Sciences, \\ 80 Nandan Road, Shanghai 200030, People's Republic of China \\ ${ }^{3}$ Xinjiang Astronomical Observatory, CAS, Urumqi, 830011, People's Republic of China \\ ${ }_{5}^{4}$ Department of Physics, Hong Kong University, Hong Kong, People's Republic of China \\ ${ }^{5}$ National Astronomical Observatories, CAS, Beijing, 100012, People's Republic of China \\ ${ }^{6}$ Key Laboratory of Radio Astronomy, Chinese Academy of Sciences, People's Republic of China \\ ${ }^{7}$ School of Astronomy and Space Science, Nanjing University, Nanjing, 210093, People's Republic of China \\ ${ }^{8}$ School of Physics and Electronic Engineering, Guangzhou, 510006, People's Republic of China \\ ${ }_{9}^{9}$ School of Physics, Peking University, Beijing 100871, People's Republic of China \\ Received 2017 October 20; revised 2018 January 16; accepted 2018 January 29; published 2018 March 5
}

\begin{abstract}
Ultra-compact pulsar binaries are both ideal sources of gravitational radiation for gravitational wave detectors and laboratories for fundamental physics. However, the shortest orbital period of all radio pulsar binaries is currently $1.6 \mathrm{hr}$. The absence of pulsar binaries with a shorter orbital period is most likely due to technique limit. This paper points out that a tidal effect occurring on pulsar binaries with a short orbital period can perturb the orbital elements and result in a significant change in orbital modulation, which dramatically reduces the sensitivity of the acceleration searching that is widely used. Here a new search is proposed. The abnormal timing residual exhibited in a single pulse observation is simulated by a tidal effect occurring on an ultra-compact binary. The reproduction of the main features represented by the sharp peaks displayed in the abnormal timing behavior suggests that pulsars like PSR B0919+06 could be a candidate for an ultra-compact binary of an orbital period of $\sim 10$ minutes and a companion star of a white dwarf star. The binary nature of such a candidate is further tested by (1) comparing the predicted long-term binary effect with decades of timing noise observed and (2) observing the optical counterpart of the expected companion star. Test (1) likely supports our model, while more observations are needed in test (2). Some interesting ultra-compact binaries could be found in the near future by applying such a new approach to other binary candidates.
\end{abstract}

Key words: binaries: general - gravitational waves - pulsars: individual (PSR B0919+06)

\section{Introduction}

As the orbital period of a pulsar binary reaches $\sim 10$ minutes, the orbital velocity of the pulsar becomes greater than that of a normal pulsar binary with an orbital period measured in hours. To search such ultra-compact binaries, the orbital modulation has to be examined at a shorter time interval (phase bin) with signals severely blurred by the orbital motion of the pulsar.

This reduces the signal-to-noise ratio of the pulse profile at each phase bin of orbit, which results in a drastic reduction in the sensitivity of pulsar searches. Various so-called acceleration searches have been developed to mitigate the loss of sensitivity caused by the orbital motion of the pulsar (Johnston \& Kulkarni 1991; Camilo et al. 2000; Ransom et al. 2003). However, no pulsar binary with an orbital period of less than 95.3 minutes (Camilo et al. 2000) has ever been observed (to date, the total number of binary pulsars is 274 according to the ATNF pulsar catalog. ${ }^{10}$ )

For a binary with an orbital period of 10 minutes and masses of the pulsar and its companion star of $M_{\mathrm{p}} \sim 1 M_{\odot}$ and $M_{\mathrm{c}} \sim 0.1 M_{\odot}$, respectively, the semimajor axis is $a=$ $\left[G\left(M_{\mathrm{p}}+M_{\mathrm{c}}\right) / n^{2}\right]^{1 / 3} \sim 10^{10} \mathrm{~cm}$. And with an orbital inclination of $\sin i \sim 0.1$ (refer to Table 1), the projected semimajor axis $x$ is typically $10^{1}-10^{2} \mathrm{~ms}$. Such an amplitude of orbital modulation should have been noticed even in a single pulse observation. However, no such pulse binary has ever been found.

\footnotetext{
${ }^{10}$ http://www.atnf.csiro.au/research/pulsar/psrcat/
}

Are there any other reasons for the reduction of sensitivity in the acceleration searching besides the Doppler effect? In fact, many acceleration searches are based on the assumption that the orbital modulation of a pulsar binary is periodic and smooth. If these searches are not periodic and smooth, which corresponds to the tidal effect we consider in this paper, we may lose some binary candidates under such search methods.

The Roemer delay, corresponding to a different propagation time than the observer as the pulsar moves at a different orbit position, is given by

$$
\Delta_{R}=x\left[(\cos U-e) \sin \omega+\left(1-e^{2}\right)^{1 / 2} \sin U \cos \omega\right],
$$

where $U$ is the eccentric anomaly, $\omega$ is the angle of the periastron, $x \equiv\left(M_{\mathrm{c}} a \sin i\right) /\left(M_{\mathrm{c}}+M_{\mathrm{p}}\right) c$ is the projected semimajor axis of the pulsar in which $a$ is the semimajor axis of the orbit, $i$ is the orbital inclination, and $c$ is the speed of light.

In this paper we point out that for an ultra-compact system of an orbital period of $\sim 10$ minutes, the separation between the two bodies, represented by the semimajor axis $a$, is about five to ten times the radius of the white dwarf (WD) star companion. In such a case, the tidal effect analogous to the planet-satellite system becomes considerable, which results in a perturbation to the orbital elements, such as $i, a, e \ldots$. These variabilities in turn lead to an additional perturbation to the Roemer delay.

Thus the arrival time of a tidal-affected binary can differ significantly from that of a normal binary pulsar approximated by two point masses neglecting the tidal effect. In other words, a normal binary pulsar with a negligible tidal effect has 


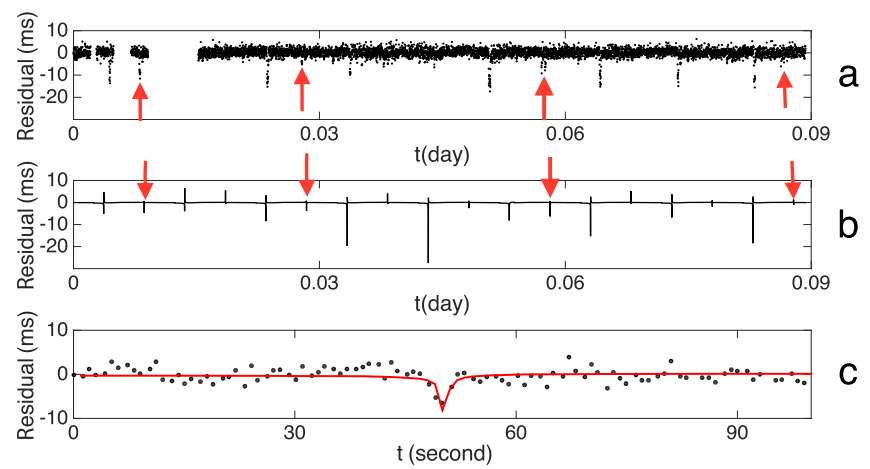

Figure 1. The time of arrival corresponds to the $2 \mathrm{hr}$ single pulse observation of PSR B0919+06. The upper panel is the observed time of arrival and the middle panel is the arrival time that corresponds to the Roemer delay perturbed by the tidal effect. The bottom panel shows a typical shift with dotted points (observed) and a continued curve (simulated), obtained by moving a simulated peak to a nearby observed one.

constant orbital elements and therefore a periodic and smooth orbital modulation, but an ultra-compact binary contaminated by the tidal effect corresponds to perturbed orbital elements and hence has a quasi-periodic and sharp orbital modulation. Such ultra-compact pulsar binaries may have been identified as singular pulsars plus abnormal timing behaviors.

A strange fluctuation of arrival time at an amplitude of $\sim 10 \mathrm{~ms}$ with a time interval of $\sim 10$ minutes was first found on the singular pulsar PSR B0919+06 (Rankin et al. 2006). The pulsation of a pulsar can be displayed as a pulse profile, with a horizontal axis of rotational phase or longitude (in degrees) and a vertical axis of flux density. Rankin et al. first noticed a strange shift in the timing residual: the pulse profile moves leftward for a few degrees (with the peak at a negative rotational longitude) in the time interval of several rotational periods of the pulsar. The pulse profile stays in this temporary position for several tens of pulses and returns to its normal state of zero rotational longitude over the span of a few pulses. The next cycle of shifts may happen in a period of a few minutes to tens of minutes.

This gradual and episodic shift of profile is sometimes described as a flare state (Perera et al. 2015), which differs from the mode-change of a pulsar in which the change of the pulse profile occurs mostly within a single pulse. It also differs from the drifting of subpulses, in which the peak usually advances in an orderly manner with longitude and then returns quickly to its original position. It cannot be caused by a sudden increase of emission altitude due to the lack of a dramatic change of position angle in the polarization pattern and/or an abrupt widening in the total emission cone (Rankin et al. 2006).

If the occasional drift events correspond to the abnormal orbital modulation of an ultra-compact binary, then a long-term observation will expect more such drifts. Therefore a continuous timing observation covering multiple orbital periods is needed. We thus observed PSR B0919+06 with the Arecibo telescope on 2014 December 26. The observation (project code P2931) lasted 114 minutes on the Puppi pulsar backend in a single-pulse mode with a sampling time of $64 \mu \mathrm{s}$. The pulse shift exhibited in such a single pulse observation can be transferred to the arrival time as shown in Figure 1(a). Though similar in individual shift to two other observations, Rankin et al. (2006) and Perera et al. (2015), this data sets show clearer and more prominent quasi-periodic features, which allows us to better investigate the underlying physics.
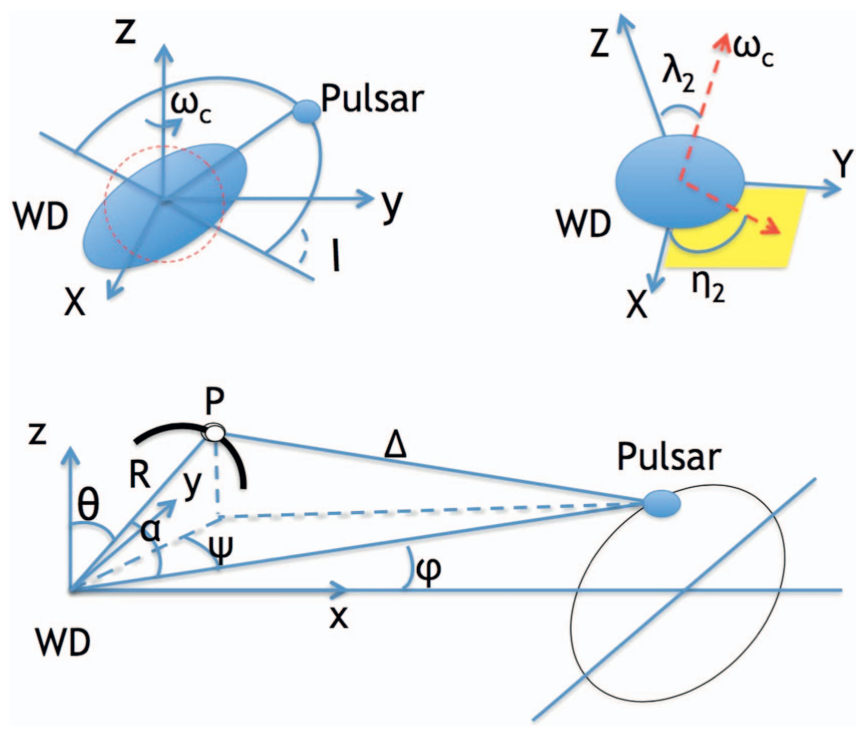

Figure 2. The geometry and parameters of the binary system under the tidal effect. Top left: the pulsar at periastron causes the most significant shape change in the WD. The orientation of the bulge is defined by the angle, $\lambda_{\mathrm{BG}}$, which is the misalignment angle between the spin axis of the WD and the line connecting the top of the bulge and the center of the WD. Top right: the misalignment angles used in the practical simulation while the orbital path is set on the $x-y$ plane. Bottom: the geometry in the coordinate frame centered on and rotating with the WD while the path of the pulsar is in the equatorial plane of the WD. Notice that point $P$ is the top of the bulge on the surface of the WD.

The observed spikes displayed in Figure 1(a) deviate from the standard Roemer delay in three aspects. First, the time interval between the two neighboring peaks (shifts) is not a constant. Second, the shifts are asymmetric about the vertical zero as shown in Figure 1(a), which corresponds to an earlier arrival time relative to that in normal cases. Third, the peak of each shift is much sharper than that of the normal orbital modulation. Whether a tidal effect occurring in an ultracompact binary can reproduce such an unexpected orbital modulation is discussed in Section 2. Section 3 tests the consistency of such an ultra-compact scenario with the longterm timing noise of this pulsar. X-ray, optical, and radio counterparts of PSR B0919+06 are also discussed. Finally, some extended discussion is made in Section 4.

\section{The Tidal Effect on an Ultra-compact Binary}

In the main-sequence star binary pulsars (with an orbital period of years), the spin-induced quadrupole momentum of the companion gives rise to the perturbation of six orbital elements of the binary system, which resemble sharp spikes at the periastron (Wex 1998). In substituting those perturbed orbital elements into the Roemer delay, the resultant timing residual at the short term also displays sharp peaks at the periastron (Wex 1998).

The spin-induced quadrupole momentum is treated as stationary in the calculation of such main-sequence star binary pulsars (Wex 1998). In contrast, for an ultra-compact binary pulsar with an orbital period of tens minutes and with a companion star of the WD, the shape of the WD varies under the tidal force exerted by the pulsar, which cannot be treated as a constant.

By a simple transformation, the perturbation of the six orbital elements obtained under a constant quadrupole momentum can be applied to the calculation of the variabilities 
Table 1

Parameters Obtained by Fitting of Single Pulse Observation

\begin{tabular}{lccccccccccc}
\hline \hline$P_{\mathrm{b}}(\mathrm{s})$ & $e$ & $M_{\mathrm{p}}\left(M_{\odot}\right)$ & $M_{\mathrm{c}}\left(M_{\odot}\right)$ & $n / \omega_{\mathrm{c}}$ & $R_{\mathrm{c}}(\mathrm{cm})$ & $M_{0}(\mathrm{rad})$ & $\lambda_{2}(\mathrm{rad})$ & $\eta_{2}(\mathrm{rad})$ & $\phi(\mathrm{rad})$ & $\lambda_{\mathrm{BG}}(\mathrm{rad})$ \\
\hline 855.5 & 0.152 & 1.15 & 0.16 & 1.37 & $3.2 \times 10^{9}$ & 5.5 & 2.0 & 4.54 & 0.85 & 1.56 \\
\hline
\end{tabular}

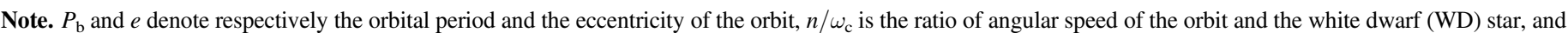

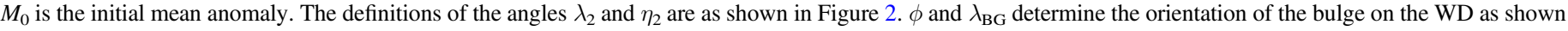
in Figure 2.

of the six orbital elements with a time-varying quadrupole momentum.

For a binary pulsar system with an orbital period of tens of minutes and a companion mass of $\sim 0.1 M_{\odot}$ (see the best-fitting parameters in Table 1), the separation between the two bodies is only about five to ten times the radius of the WD companion. In such a circumstance, the tidal force on the WD is significant enough to give rise to a tidal bulge on the WD, which can be described by the parameter $\epsilon_{2}$,

$$
R(\beta)=R_{\mathrm{c}}\left[1+\epsilon_{2} P_{2}(\cos \beta)\right],
$$

where $P_{2}(\cos \beta)=(1 / 4)(3 \cos 2 \beta+1)$ is the Legendre polynomial of degree $2, R_{\mathrm{c}}$ is the WD radius, and $\beta$ is the misalignment angle between the axis of symmetry of the bulge and a reference axis. Apparently, such a bulge varies with orbital phase, which corresponds to a time-varying quadrupole momentum.

As estimated in Appendix A, the additional potential corresponds to an additional force on the WD, which corresponds to the torque $\Gamma$. Finally, the torque can change the shape of the bulge described by Equation (2), which corresponds to a time-varying quadrupole momentum of the WD, as discussed in detail in Appendix A.

The numerical result of such a time-varying quadrupole momentum on the arrival time of an ultra-compact binary can be obtained by a constant quadrupole momentum (Wex 1998), which is represented by a constant dimensionless parameter, $Q$ (say $Q=Q_{1}$ ). The parameter $Q$ is defined by the ratio of the tidal potential and the gravitational potential from the pulsar, which is read as $Q=V_{\mathrm{q}} / V_{\mathrm{PSR}} \approx \frac{3}{2} R^{2} / a^{2}$, where $R$ is given by Equation (2) and $V_{\mathrm{PSR}}$ and $V_{\mathrm{q}}$ are given in Appendix A.

Such a $Q$ gives rise to an additional potential of gravity in the dynamics of the two-body system, which results in the perturbation of the six orbital elements. And in turn the variation of these orbital elements causes additional variabilities in the Roemer delay that can appear as sharp peaks at the periastron (Wex 1998).

In the case of a constant $Q$, the perturbation of an orbital element, e.g., semimajor axis are $\Delta a_{1}\left(t_{1}\right)$ and $\Delta a_{1}\left(t_{2}\right)$ at two neighboring times, $t_{1}$ and $t_{2}$, respectively.

In contrast, as $Q$ varies with time, e.g., at times $t_{1}$ and $t_{2}$, the value of $Q$ is $Q_{1}$ and $Q_{2}$, respectively. Then the perturbation of an orbital element, e.g., the semimajor axis, at time $t_{1}$ and $t_{2}$ must be calculated under two different $Q s$ : (1) at time $t_{1}$ with $Q=Q_{1}$, resulting in $\Delta a_{1}\left(t_{1}\right)$, and (2) at time $t_{2}$ with $Q=Q_{2}$, resulting in $\Delta a_{2}\left(t_{2}\right)$.

In other words, at times $t_{1}$ and $t_{2}$, a constant $Q$ gives an element perturbation of $\Delta a_{1}\left(t_{1}\right)$ and $\Delta a_{1}\left(t_{2}\right)$, respectively; in comparison, a variable $Q$ predicts $\Delta a_{1}\left(t_{1}\right)$ and $\Delta a_{2}\left(t_{2}\right)$. By substituting these perturbations of the six orbital elements under a variable $Q$ into the Roemer delay of Equation (1), the tidal effect-induced timing residual of PSR B0919+06 can be obtained numerically.
From the point of view of the perturbation theory of the dynamics of a two-body system, no matter whether the quadrupole momentum is fixed or time varying, the corresponding additional potential cannot influence the dynamics of the two-body system of two point masses. This condition is well satisfied because $V_{\mathrm{q}} / V_{\mathrm{PSR}} \approx \frac{3}{2} R_{\mathrm{c}}^{2} / a^{2} \approx 1 \times 10^{-2}$ by the parameter as shown in Table 1 , which corresponds to a variation in the Roemer delay of an amplitude of $\sim 10 \mathrm{~ms}$.

The best numerical fit reproducing the three observational characters with the smallest deviation is as shown in Figure 1(b), which corresponds to the fitting parameters of Table 1. The superposition of such a predicted orbital modulation of a $10 \mathrm{~ms}$ amplitude with dense data points at a $5 \mathrm{~ms}$ level originating from another source of noise explains the observation of Figure 1(a).

The Monte Carlo method is applied in the simulation of the observed single pulse observation as shown in Figure 1(a). With the initial values of the binary parameters given by fitting the long-term timing noise as shown in Table 2, each fitting parameter is searched in a wide space in order to find the best combination of parameters so that the differences between the observed and simulated spikes, both the feature of an individual spike and the relative positions among them, can be minimized.

Comparing the observations of Figure 1(a) and the best simulation of Figure 1(b), the features of asymmetry and sharpness exhibited in the spikes can be well fitted by the tidal effect. However the quasi-periodicity display in the observation cannot be well reproduced by the model. In other words, the positions of the observed and simulated shift peaks have considerable differences - the average discrepancy between the observed and the simulated peaks is $100 \mathrm{~s}$ (equivalent to $12 \%$ of the orbital period), as shown in Figure 1. In the simulation, the dominant deviation between the observed and the simulated data is contributed by the position difference.

In the simulation of Figure 1, the deformation of the WD (denoted by the quadrupole momentum as shown in Appendix B) is assumed to be proportional to the external torque, $Q(t) \propto \Gamma(t)$. In practice, the response of $Q(t)$ to $\Gamma(t)$ is more complicated, depending on the internal density distribution, elasticity, viscosity, tidal heat, etc., which can deviate from a linear relationship. Further improvement in the fitting depends on the progress in the field of the internal properties of the WD, which will be investigated in separate works.

Nevertheless, the main features displayed in the single pulse observations of PSR B0919+06 are reproduced for the first time with reasonable consistency. It is interesting that the tidal effect results in sharp spikes in the timing residual in both a wide binary with an orbital period of years (Wex 1998) and an ultra-compact binary of an orbital period of $\sim 10$ minutes. No wonder it appears so puzzling - it can be attributed to neither the mode change nor the drifting of the subpulse.

As shown by the red arrows in Figure 1(b), a relatively small shift in the middle of each period is expected, which 
Table 2

Parameters Obtained by Fitting the Long-term Timing Noise ${ }^{\mathrm{a}}$

\begin{tabular}{lcccccccc}
\hline \hline$P_{\mathrm{b}}{ }^{*}(\mathrm{hr})$ & $M_{\mathrm{c}} / M_{\mathrm{p}}$ & $I(\mathrm{rad})$ & $c_{\mathrm{c}}$ & $\eta_{10}(\mathrm{rad})$ & $\eta_{20}(\mathrm{rad})$ & $\lambda_{1}(\mathrm{rad})$ & $\lambda_{2}(\mathrm{rad})$ & $\phi_{0}(\mathrm{rad})$ \\
\hline $0.262 \pm 0.002^{\mathrm{a}}$ & $0.130 \pm 0.001$ & $3.110 \pm 0.002$ & $0.0047_{-0.0014}^{+0.0018}$ & $-0.46 \pm 0.13$ & $2.58_{-1.09}^{+1.11}$ & $3.095_{-0.007}^{+0.002}$ & $0.86_{-0.33}^{+0.41}$ & $-0.009 \pm 0.01$ \\
\hline
\end{tabular}

Note. $P_{\mathrm{b}}{ }^{*}$ is defined in Equation (8). $I$ is the misalignment angle between our line of sight and the total angular momentum vector (orbtial angular momentum). $c_{\mathrm{p}}=S_{\mathrm{p}} / L$ and $c_{\mathrm{c}}=S_{\mathrm{c}} / L$ are the dimensionless spin angular momentum for the pulsar and the companion. $\eta_{10}, \eta_{20}, \lambda_{1}$ and $\lambda_{2}$ describe the geometric configuration for two spin vectors. $\phi_{0}$ is the initial phase for $\Theta(t)$. For details of the model, we refer the readers to Gong $\& \mathrm{Li}(2013)$. $c_{\mathrm{p}}$ is not included in the sampling since our model is weakly dependent on it $\left(c_{\mathrm{p}} \lesssim 0.01\right)$.

${ }^{\mathrm{a}}$ The errors are all for a $1 \sigma$ confidence limit.

corresponds to a variation frequency of $2 / P_{\mathrm{b}}$. This predicted tidal shift is analogous to the fortnightly tide in the EarthMoon system. The signature of such small shifts between two main shifts (corresponds to $P_{\mathrm{b}}$ ) is noticeable as shown by the red arrows in Figure 1(a). Further observation is still needed to confirm such a subtle effect. At any rate, Figure 1(a) provides the first possible evidence of the existence of such subtle shifts of this pulsar although major shifts have been observed extensively (Han et al. 2016; Wahl et al. 2016).

Consequently, with the binary parameters of Table 1 the orbital modulation with a tidal effect can reproduce asymmetric and sharp peaks displayed in a single pulse observation. The quasi-periodicity and the signature of a fortnightly tide still need further theoretical and observational investigation for confirmation.

\section{Long-term Timing Noise, X-Ray, and Optical Observations}

The scenario of the ultra-compact binary of PSR B0919+06 should have a long-term effect that can be compared with the longterm timing noise of this pulsar. By the orbital parameters predicted by Table 1, such a candidate of a pulsar binary with an orbital period of 14 minutes corresponds to a geodetic precession of periods of years and 10 years for the WD and the pulsar, respectively. Indeed, the long-term timing noise of a timescale of 30 years displays a quasi-periodic modulation of a timescale of 600 days and 10 years, respectively (Shabanova 2010; Perera et al. 2015).

Such a quasi-periodic feature of timing noise may originate in the geodetic precession of the pulsar spin axis under the gravitational field of its companion. The precession is equivalent to a "spinning up" and "spinning down" of the pulsar spin, which leads to an additional delay in the arrival of pulses.

Fitting the observed timing noise (Lyne et al. 2010; Shabanova 2010) by the precession-induced timing delay (Gong \& Li 2013; see also Appendix B for details), we can obtain the orbital parameters from the Markov Chain Monte Carlo (MCMC) method with their best-fit values and $1 \sigma$ uncertainties listed in Table 2.

From Table 2, the misalignment angle between the spin axis of the pulsar and the orbital plane is $\lambda_{1}=3.10$, which means the precession cone of the pulsar spin axis has an opening angle of only $2^{\circ} .6$. Therefore, the precession of the pulsar spin will cause a very limited change in the spin axis with respect to the line of sight, so that the pulsar has been observed for decades.
By the binary parameters of Table 1, the Roche lobe radius of the WD (Frank et al. 2002) is given as

$$
R_{\mathrm{L}}=0.462 a\left(\frac{m_{\mathrm{c}}}{m_{\mathrm{p}}+m_{\mathrm{c}}}\right)^{1 / 3}=3.4 \times 10^{9} \mathrm{~cm},
$$

which is a little larger than the WD radius of $R_{\mathrm{c}} \approx 3.2 \times 10^{9} \mathrm{~cm}$ as shown in Table 1, obtained by the simulation of Figure 1(a). As analyzed in Section 2, such an $R_{\mathrm{c}}$ given by Table 1 is calculated by assuming the WD as a sphere plus two bulges changing linearly with the tidal force. In practice the shape of the WD may be more complicated and the response of the WD shape to tidal force is most likely nonlinear.

A WD with a mass of $0.16 M_{\odot}$ will have a radius of at least $\sim 1.5 \times 10^{9} \mathrm{~cm}$, depending on the exact composition and entropy based on the zero-temperature equation (Verbunt \& Rappaport 1998; Marsh \& Nelemens 2004), which could be enlarged further by tidal heating or irradiation. Regardless, the $R_{\mathrm{c}}$ shown in Table 1 can be treated as a simplification to a more complicated configuration.

Although on average the radius of the WD is less than its Roche lobe radius, $R_{\mathrm{c}}<R_{L}$, according to Equation (3) at the periastron the Roche lobe radius of the WD can be reduced up to $R_{\mathrm{L}} \approx 2.8 \times 10^{9} \mathrm{~cm}$, which is $10 \%$ less than the WD radius. In the orbital phase neighboring the passage of the periastron, $R_{\mathrm{c}}>R_{L}$ can be satisfied so that the WD can fill the Roche lobe and hence mass transfer occurs.

However, such a possibility is restricted by three factors. First, if the WD expands less rapidly or shrinks faster than its Roche lobe, then the mass transfer near the periastron will be limited. As discussed in Section 2, the response of the WD shape to tidal force is most likely nonlinear, which may prevent the condition of $R_{\mathrm{c}}>R_{L}$ near the periastron from happening.

Second, the situation when the radius of the secondary follows the evolution of the secondary Roche lobe radius, $\dot{R}_{\mathrm{c}} / R_{\mathrm{c}}=\dot{R}_{L} / R_{L}$, has been addressed in the evolution of a binary pulsar system (Di Salvo et al. 2008). If this is the case for the change in the WD radius and Roche lobe radius of PSR B0919+06, then $R_{\mathrm{c}}<R_{L}$ can be satisfied in all orbital phases.

Third, even if a certain mass transfer occurs on the WD, such matter cannot fall onto the surface of the pulsar due to the propeller effect as, in the following discussion.

The X-ray luminosity of PSR B0919+06 observed by the $X M M$-Newton telescope is only (3-5) $\times 10^{30} \mathrm{erg} \mathrm{s}^{-1}$, as shown in Appendix C. In comparison, the accreting millisecond pulsar SAX J1808.4-3658 has an X-ray luminosity as high as $(4.7-6.4) \times 10^{35} \mathrm{erg} \mathrm{s}^{-1}$ during an outburst (e.g., Hartman et al. 2008), in which accreted matter is thought to be transferred onto the surface of the pulsar (Di Salvo et al. 2008). The deviation of 
luminosity between PSR B0919+06 and SAX J1808.4-3658 suggests that the accretion process is likely suppressed in PSR B0919+06.

For a binary with parameters listed in Table 1 (assuming a mass-radius index of $-1 / 3$ ), the predicted mass loss rate from the companion star, driven by the angular momentum loss due to gravitational radiation, could be $3 \times 10^{-8} \sim$ $1 \times 10^{-7} M_{\odot} \mathrm{yr}^{-1}$ depending on whether the mass transfer is conservative (all mass loss is accreted by the pulsar) or nonconservative (e.g., almost all mass loss is transferred to the wind) (Di Salvo et al. 2008).

The corotation radius of PSR B0919+06 is given by

$$
r_{\mathrm{co}}=\left(\frac{G M_{\mathrm{p}} P^{2}}{4 \pi^{2}}\right)^{1 / 3}=9 \times 10^{7} \mathrm{~cm} .
$$

The corresponding accretion rate is $\dot{M} \sim 6 \times 10^{-14} M_{\odot} \mathrm{yr}^{-1}$, estimated from $L_{\mathrm{x}}=G m \dot{M} /\left(2 r_{\mathrm{co}}\right)$, with $L_{\mathrm{x}} \approx 4 \times 10^{30} \mathrm{erg} \mathrm{s}^{-1}$. This value is significantly lower than the inferred values from the mass transfer rate driven by the gravitational radiation losses noted above. This could be due to the suppression of the accretion process, apart from the mass loss carried away by the wind.

With a pulsar radius of $10 \mathrm{~km}$ and a spindown magnetic field of a $2.5 \times 10^{12} \mathrm{G}$ (given by $P$ and $\dot{P}$ ) strength, the magnetosphere radius of the pulsar is given by a balance of an average magnetic pressure with the ram pressure of a spherical accretion flow (Lamb \& Pethick 1973; Cui 1997):

$$
\begin{aligned}
R_{\mathrm{m}}= & 2.7 \times 10^{8}\left(\frac{L_{\mathrm{x}}}{10^{37} \mathrm{erg} \mathrm{s}^{-1}}\right)^{-2 / 7}\left(\frac{M_{\mathrm{p}}}{1.4 M_{\odot}}\right)^{1 / 7} \\
& \times\left(\frac{B}{10^{12} \mathrm{G}}\right)^{4 / 7}\left(\frac{R}{10^{6} \mathrm{~cm}}\right)^{10 / 7} \mathrm{~cm} \\
= & 3 \times 10^{10} \mathrm{~cm} .
\end{aligned}
$$

By the estimations of Equations (5) and (4), the magnetosphere radius of PSR B0919+06 is much larger than its corotation radius.

The magnetosphere of a pulsar is normally a dipole field configuration, where the maximum strength is along the magnetic axis. For PSR B0919+06, as shown above Equation (3), the misalignment angle between the spin axis of the pulsar and the orbital plane is only $2^{\circ} .6$. Considering a magnetic inclination angle of a few degrees, the misalignment angle between the magnetic axis and the orbital plane is also of a few degrees. In other words, the magnetic axis of PSR B0919+06 is close to the line of sight and its orbital plane is nearly face on. Therefore, the strength of the magnetic field of PSR B0919+06 is strongest near the line of sight and weakest near the orbital plane. Correspondingly the practical magnetosphere radius is largest $\left(\sim 10^{10} \mathrm{~cm}\right)$ near the line of sight and shortest $\left(\sim 10^{9} \mathrm{~cm}\right)$ near the orbital plane, which is still much larger than the corotation radius.

With such a dipole field configuration, the relationship among the corotation radius, the magnetosphere radius, and the light cylinder radius can satisfy $r_{\mathrm{co}}<R_{\mathrm{m}}{ }^{\prime}<r_{\mathrm{L}}$ (where $r_{\mathrm{L}}=2.1 \times 10^{9} \mathrm{~cm}$ is the light cylinder radius).

In this case, the centrifugal force prevents the accreting material from reaching the magnetic pole and the pulsar is in the so-called propeller phase, in which material is presumably ejected away from the pulsar corresponding to a slowing down of the pulsar spin frequency (Illarionov \& Sunyaev 1975).
Observational evidence of the propeller phase has been found on X-ray pulsars. The total X-ray flux of these pulsars dropped significantly, which indicated a decrease in the accretion rate and increase in the magnetosphere radius (Cui 1997; Zhang 1998).

Interestingly, the spindown luminosity of PSR B0919+06 is $L_{\mathrm{rot}} \approx 7 \times 10^{33} \mathrm{erg} \mathrm{s}^{-1}$, which is three orders of magnitude higher than its X-ray luminosity. Such a luminosity deviation may originate in anisotropy of radiation, additional heating, uncertainty in distance, and instrument limitations (Shibata et al. 2016).

Apparently the discrepancy between $L_{\mathrm{rot}}$ and $L_{\mathrm{x}}$ can be explained by the scenario of suppressed accretion as the pulsar in the propeller phase, which is indicated by the small corotation radius of Equation (4) compared with the magnetosphere radius of Equation (5) (even taking into account the dipole field configuration).

This scenario of the propeller phase for B0919+06 is supported by the model for the distribution of free electrons in the Galaxy, the Magellanic Clouds, and the intergalactic medium, which can be used to estimate distances for real or simulated pulsars based on their dispersion measure (Yao et al. 2017). This model can show a distance of $1.9 \mathrm{kpc}$ from PSR B0919+06 while parallax measurement by the long baseline observations shows a distance of between 1.0 and $1.3 \mathrm{kpc}$.

Taking the $1.2 \mathrm{kpc}$ distance of the long baseline observation of Chatterjee et al. (2001), the distance discrepancy between the approach of parallax (Chatterjee et al. 2001) and the distribution of free electrons (Yao et al. 2017) is $\Delta L=0.7 \mathrm{kpc}$. Assuming the scale of plasma distribution of PSR B0919+06 is $\Delta L^{\prime} \sim 10^{10} \mathrm{~cm}$, then the plasma density surrounding PSR B0919+06 can be estimated by $n_{\mathrm{e}}^{\prime}=n_{\mathrm{e}} \Delta L / \delta L^{\prime} \sim 10^{9} n_{\mathrm{e}}$, where $n_{\mathrm{e}}$ denotes the average electron density in the direction of PSR B0919+06. Such a high density of plasma may result in the propeller effect occurring in PSR B0919+06.

To test the expected WD counterpart of PSR B0919+06, we observed PSR B0919+06 with the $4.2 \mathrm{~m}$ William Hershel telescope (WHT) in 2016 by the Sloan Digital Sky Survey (SDSS) $u g r$ band.

Assuming that the cooling time of the WD equals the characteristic age of PSR B0919+06, 0.5 Myr (Shabanova et al. 2013), the luminosity of the WD can be derived from the WD cooling model (Althaus \& Benvenuto 1997). Knowing that the distance of the pulsar is $1.21 \mathrm{kpc}$ (Chatterjee et al. 2001), the apparent magnitude of the WD is estimated as $~ 25$ mag.

A possible optical counterpart (hereafter WHT1) was detected with magnitudes of $u=25.68(0.30), g=25.25(0.22)$, and $r=25.10(0.13)$. The dereddened colors $(u-g)_{0}$ and $(g-r)_{0}$ of WHT1 are consistent with hot WDs in the $u-g$ versus $g-r$ color diagram as shown in Figure 3.

However, the position observation of PSR B0919+06 is complicated. The position and proper motion of PSR B0919 +06 have been measured by very long baseline array (Chatterjee et al. 2001) and by very large array (Brisken et al. 2003). Pulsar timing also gives a position in radio (Hobbs et al. 2004). The X-ray position was observed by an XMMNewton proposal (ID 05029201) in 2007, and the source is compiled in the XMM-Newton Serendipitous Source Catalog (Rosen et al. 2016). 


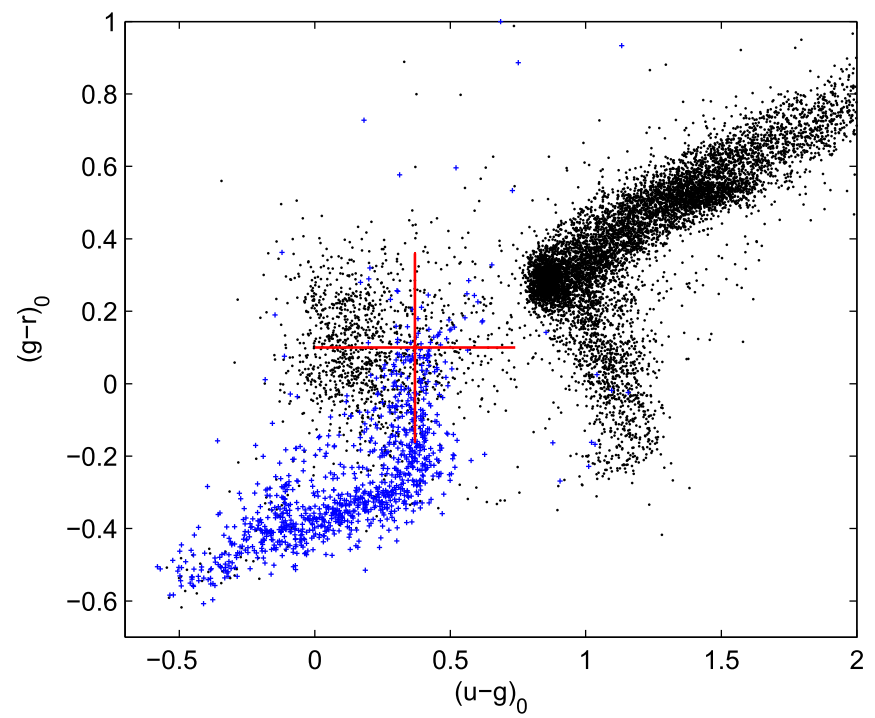

Figure 3. The $(u-g)_{0}$ vs. $(g-r)_{0}$ diagram of a sample of SDSS point sources as well as the colors of WHT1. The dereddened colors are calculated using extinctions of nearby quasi-stellar objects (QSOs) in the sky. The blue dots are WDs. The small black dots are nondegenerate stars and QSOs. The bulk of black dots to the right are nondegenerate stars, and those clustered near the WDs are QSOs, which can be distinguished from the WDs by radio observations. The large red cross denotes WHT1 and the errors of its colors. WHT1 is located directly in the area of the WDs.

To compare the various positions given by different observations, we used the proper motion of Chatterjee et al. (2001) to convert positions at different epochs to the same epoch when our latest optical observation was carried out.

As shown in Figure 4, the converted positions are presented as triangles in different colors. The $1 \sigma$ error eclipses of positions are plotted in solid line and the $2 \sigma$ error eclipses are in dashed line. The error eclipses of the radio positions obtained from three measurements (Chatterjee et al. 2001; Brisken et al. 2003; Hobbs et al. 2004) are much smaller than those of the X-ray and optical counterparts, so they are shown with a zoom-in in the inset. The error eclipse of the Chatterjee et al. (2001) position is so small that it cannot be shown even zoomed in.

Apparently, the positions given by long baseline observation, pulsar timing, X-ray observation, and optical observation are not consistent with each other, as shown in Figure 4.

The position offsets between different radio observations can be as large as $\sim 1^{\prime \prime}$, although they have a very high accuracy. Considering the large proper motion of the pulsar, a small error in it can result in deviations between the positions measured at different epochs after the correction of such a proper motion. Since the radio observations were performed more than 15 years ago, the radio-optical offset of $2^{\prime \prime}-3^{\prime \prime}$ cannot rule out that WHT1 is the optical counterpart of PSR B0919+06. Multiwavelength observations of the counterpart of PSR B0919 +06 are expected to confirm the position in the future.

\section{Discussion}

Consequently, with the binary parameters of Table 1, the orbital modulation features with the tidal effect are consistent with asymmetric and sharp peaks displayed in a single pulse observation. In addition, the long-term timing noise can be explained by the geodetic precession effect in an ultracompact binary system with similar orbital parameters. The quasi-periodicity, the signature of a fortnightly tide, and optical, X-ray, and gamma-ray counterparts require future

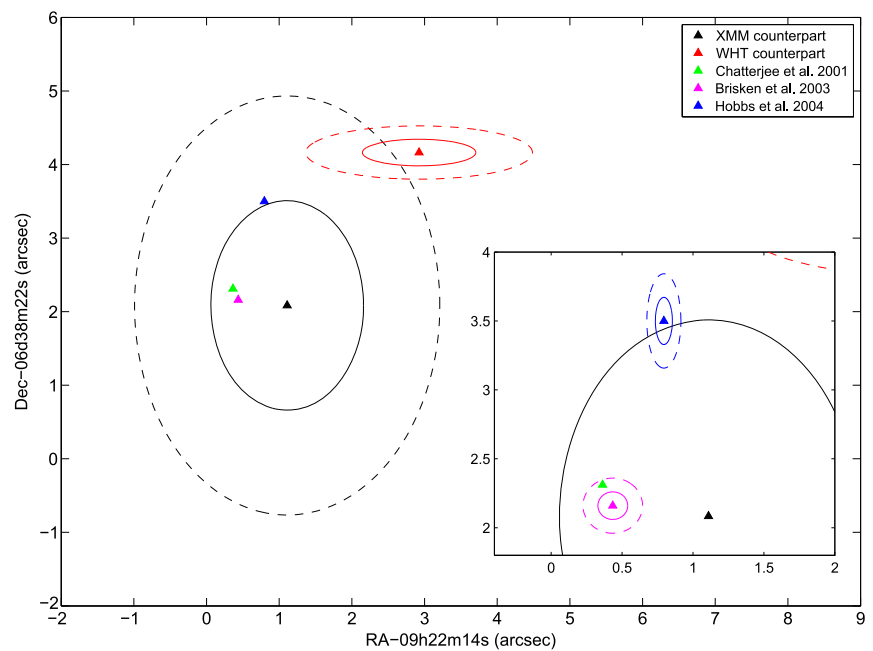

Figure 4. Radio, optical and X-ray measurements of the position of PSR B0919+06. To compare the positions of various observations, the proper motion of Chatterjee et al. (2001) was utilized to convert positions at different epochs to the same epoch when the latest optical observation was carried out. The converted positions are presented as triangles in different colors. The $1 \sigma$ error eclipses of positions are plotted in solid line and the $2 \sigma$ error eclipses are in dashed line. The error eclipses of the radio positions obtained from these three measurements are much smaller than those of the X-ray and optical counterparts, so they are shown with a zoom-in in the inset. The error eclipse of the Chatterjee et al. (2001) position is so small that it cannot be shown even by zooming in.

confirmation. A direct observation of the expected WD spectrum by large optical telescopes may provide strong support to the scenario of an ultra-compact binary to PSR B0919+06.

The long-term timing noise is better fitted than that of the single pulse observation, because the physics determining the long-term behavior is relatively simpler than that of the short-term.

In the simulation of the tidal effect, one can ignore the effect of general relativity for the following reasons. The short-term effect of general relativity (at the timescale of an orbital period) is the Einstein delay (which is of the order of $1 / c^{2}$ ) and the Shapiro delay (which is of the order of $1 / c^{3}$ ). By the fitted orbital parameters of Table 1, the amplitude of the Einstein delay of the ultra-compact binary is only $37 \mu \mathrm{s}$, which is $2-3$ orders of magnitude less than that of the $10 \mathrm{~ms}$ amplitude of shift discussed in our paper, so the effect of general relativity in the short term can be safely disregarded.

As shown in Appendix B, the torque of the pulsar exerted on the WD companion is $\Gamma \propto R_{\mathrm{c}}^{2} / a^{3} \propto R_{\mathrm{c}}^{2} / P_{\mathrm{b}}^{2}$. For the double pulsar, PSR J0737-3039A/B of a $2.4 \mathrm{hr}$ orbital period and a pulsar companion with a $15 \mathrm{~km}$ radius, the difference between the torque on PSR B0919+06 and on PSR J0737-3039A/B is $\Gamma_{09019} / \Gamma_{0737} \approx 2 \times 10^{11}$. Therefore the tidal effect on an ultra-compact binary of the WD companion is much stronger than on a normal pulsar binary, such that the orbital modulations of the former deviate significantly from those of the latter.

The formation of such a binary is extremely difficult (or impossible) through standard channels, so there must be some alternative methods to form this binary. For example, it could originate from the merger of double WDs. While a lower-mass WD is tidally disrupted, part of it merges with a more massive WD to be a neutron star, and the other part is ejected but still bound to the newborn neutron star, forming a close binary. 
With the orbital parameters as shown in Table 1, the coalescing time of the ultra-compact binary can be estimated by

$$
\frac{\dot{P}_{\mathrm{b}}}{P_{\mathrm{b}}}=-\frac{96}{5} \frac{G^{3}}{c^{5}} \frac{M^{2} \mu}{a^{4}} f(e),
$$

where $f(e)=\left(1+\frac{73}{24} e^{2}+\frac{37}{96} e^{4}\right)\left(1-e^{2}\right)^{-7 / 2}$ and $\mu$ is the reduced mass. According to Equation (6), the lifetime of the binary via gravitational radiation is estimated at $P_{\mathrm{b}} / \dot{P}_{\mathrm{b}} \approx$ 2.9 million years, which provides a new source of gravitational waves other than neutron star-neutron star and WD-WD binaries.

We thank D. Lai, B. W. Stappers, A. Lyne, J. M. Rankin, J. Weisberg, J. Hobbs, R. N. Manchester, K. J. Lee, Q. H. Peng, and R. X. Xu for helpful discussions and suggestions. This research is supported by the National Natural Science Foundation of China under grant Nos. NSFC11373018, NSFC 11703064, and NSFC11503006, and Beyond the Horizons 2012, and the support of Strategic priority Research Program, grant No. XDB23010200.

\section{Appendix A \\ The Precession Velocity of the Binary System}

How does the precession of the spin axis result in quasiperiodic change in the timing noise and pulse profile variability? In the gravitational two-body system, due to the curvature of spacetime produced by the companion star, the rotation axis of a freely falling object suffers a precession with respect to a distant observer; this effect is known as geodetic precession.

The spin angular momentum vector of one body precesses around the orbital angular momentum vector with velocity, given by the two-body equation (Barker \& O'Connell 1975):

$$
\begin{array}{r}
\dot{\Omega}_{\mathrm{p}}=\frac{L\left(4+3 m_{\mathrm{c}} / m_{\mathrm{p}}\right)}{2 r^{3}} \hat{\boldsymbol{L}} \\
=T_{\odot}^{2 / 3}\left(\frac{2 \pi}{P_{\mathrm{b}}^{*}}\right)^{5 / 3} \times \frac{M_{\mathrm{r}}\left(4+3 M_{\mathrm{r}}\right)}{2\left(1+M_{\mathrm{r}}\right)^{4 / 3}} \hat{\boldsymbol{L}},
\end{array}
$$

where $L$ and $\hat{\boldsymbol{L}}$ are the magnitude and unit vector of the orbital angular momentum, $m_{\mathrm{p}}$ and $m_{\mathrm{c}}$ are the masses of the pulsar and its companion respectively, and $r$ is the separation between them. The rest parameters in Equation (7) are defined by $M_{\mathrm{r}} \equiv m_{\mathrm{c}} / m_{\mathrm{p}}, T_{\odot} \equiv G M_{\odot} / c^{3}=4.925490947 \mu \mathrm{s}$, and

$$
P_{\mathrm{b}}^{*} \equiv P_{\mathrm{b}}\left(1-e^{2}\right)^{\frac{3}{5}} /\left(m_{\mathrm{p}}^{*}\right)^{\frac{2}{5}}
$$

where $m_{\mathrm{p}}^{*} \equiv m_{\mathrm{p}} / M_{\odot}, P_{\mathrm{b}}$ and $e$ are the orbital period and eccentricity, respectively. Notice that the precession velocity of the WD, $\dot{\Omega}_{\mathrm{c}}$, can be obtained by replacing $M_{\mathrm{r}}$ of Equation (7) with $1 / M_{\mathrm{r}}$.

The geodetic precession can change the orientation of the spin axis and thus affects the azimuth and the latitude at which the observer's line of sight crosses the beam. This in turn leads to an additional time delay of the arrival of the pulsar and the pulse profile, respectively. This model explains not only the quasi-periodic feature as shown Figure 1, but also the correlation between the timing behavior and the variation of the averaged pulse shape (Lyne et al. 2010; Gong \& Li 2013).

The oscillation timescale of 600 days and 10 years corresponds to the precession period of the WD and the pulsar, respectively. The precession of these two stars determines the sum of the spin angular momenta of the binary, which couples with the orbital angular momentum by the conservation of the total angular momentum (sum of spin and orbit). Therefore, the precession of the two stars of the binary system affects the precession of the orbital angular momentum, which in turn affects the arrival time of the pulsar in orbital motion via the change in the orbital inclination angle. A detailed formula of such a precession-induced time delay can be found in Gong \& Li (2013).

An MCMC method is adopted to fit the long-term timing residual data collected from Lyne et al. (2010) and Shabanova (2010) based on the precession model (Gong \& Li 2013). We assume a uniform prior for all model parameters and conduct sampling with a classical Metropolis-Hastings algorithm based on the posterior probability distributions calculated by Bayesian inference. The one- and two-dimensional (1D and 2D) probability distributions of the parameters are shown in Figure 5. The mean values, indicated as the blue lines in Figure 5, and the $1 \sigma$ uncertainties of the parameters, are listed in Table 2. Note that the parameter $c_{\mathrm{p}}$ is removed because of its rather weak model dependence.

We quantify the goodness of fit from $\chi_{\nu}^{2}=\chi^{2} /$ $(N-m)=1.3$ with $N=221$ and $m=9$ being the data point and number of model parameters and assuming that the error bar for all data points is $\sigma_{i}=10 \mathrm{~ms}{ }^{11}$ This large $\chi_{\nu}^{2}$ is mainly contributed by the discrepancy in the left sharp decline of several data points. It is shown that most parameters can be converged reliably and be constrained very well, except for $\lambda_{2}$, which displays an extended tail in the 1D probability distribution in Figure 5. More important, the main orbital parameters (e.g., $P_{\mathrm{b}}^{*}, M_{\mathrm{r}}$ ) obtained here are roughly consistent with those constrained from the tidal effect as shown in Table 1.

Based on the parameters in Table 1, the long-term timing noise can be modeled as shown in Figure 6 . We can see that there is still a discrepancy between the observed and the calculated timing residual (see the lower panel of Figure 6); this most likely originates in the negligence of the quadrupole moment-induced precession of the WD. How to consider the long-term effect of a variable quadrupole moment needs further investigation.

\section{Appendix B}

\section{The Tide Potential, Torque, and Evolution Scenario}

The tide potential, force, and torque can be calculated in the coordinate system analogous to the tidal force in the planetsatellite system (Murray \& Dermott 1999).

The gravitational potential exerted on the WD (with bulge) by the pulsar is given by

$$
V_{\mathrm{PSR}}=-G \frac{M_{\mathrm{p}}}{\Delta},
$$

where $\Delta$ is the distance from an arbitrary point on the surface of the WD with a position vector, $\boldsymbol{P}$, to the pulsar center as shown in the bottom of Figure 2.

As also shown in the bottom of Figure 2, the coordinate frame is centered on and rotating with the WD (the pulsar moves in the equatorial plane of the WD), in which the trajectory of the pulsar is an ellipse about its guiding center.

\footnotetext{
11 The adoption of the error bar, although relatively large compared to the timing analysis, is to mimic the model uncertainty arising from the tidal effect, which is not considered in our long-term timing noise modeling. This assumption could influence the error estimation of model parameters but should not significantly influence their best-fit values.
} 


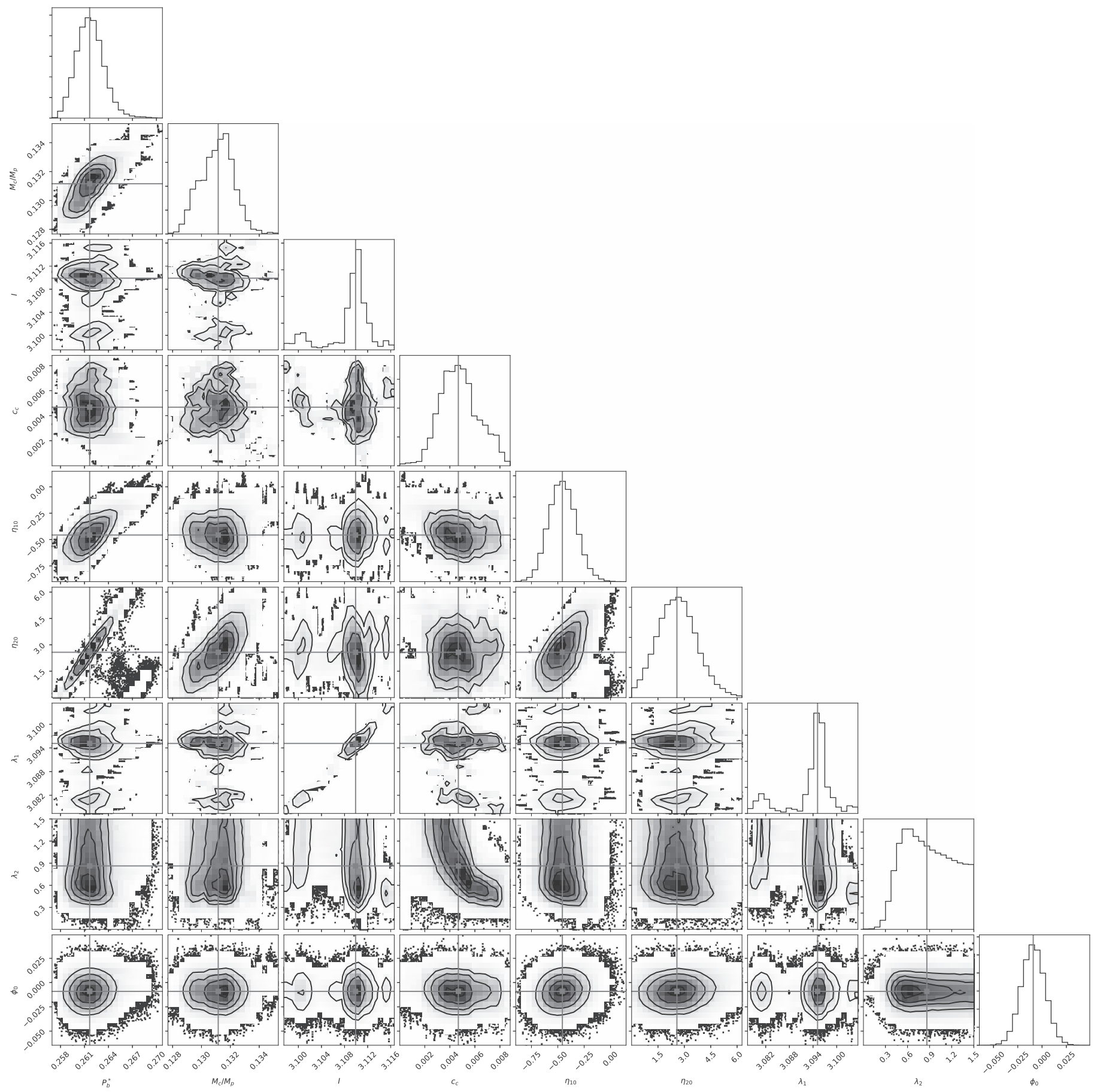

Figure 5. 1D and 2D probability distributions of the fitting parameters from our precession model. The contours in the 2D plots are for confidence levelsof $68 \%, 95 \%$, and $99.7 \%$ from inside to outside, respectively. The blue lines represent the adopted best-fit values for each parameters.

Also shown in the bottom of Figure 2, $\psi$ is the misalignment angle between the projection of the position vector $\boldsymbol{P}$ onto the $X-Y$ plane and the WD-neutron star line, which is given by $\psi=\phi-\varphi=\phi-2 e \sin n t$, with $\phi$ denoting the longitude of $P$. As shown in the bottom of Figure 2, the angles $\alpha, \theta$, and $\psi$ are related by (Murray \& Dermott 1999)

$$
\cos \alpha=\sin \theta \cos \psi \approx \sin \theta \cos (\phi-2 e \sin n t) .
$$

Rewriting the $\Delta$ of Equation (9) of the main text by the angles as shown by the geometry of Figure 2, the tidal potential of the WD becomes (Murray \& Dermott 1999)

$$
\begin{aligned}
V_{\mathrm{q}}=-G \frac{M_{\mathrm{p}}}{a} \frac{R_{\mathrm{c}}^{2}}{a^{2}}\left[P_{2}(\cos \beta)+3 e P_{2}(\cos \beta) \cos n t+\right. \\
\left.3 e \sin ^{2} \theta \cos 2 \phi \sin n t\right],
\end{aligned}
$$

where $\beta$ is the misalignment angle between the position vector $\boldsymbol{P}$ and the line joining the center of the WD and the guiding center of the pulsar's orbit, which is related with the other two angles by $\cos \beta=\cos \theta \cos \phi$, where $\theta$ and $\phi$ are defined in Figure 2. 


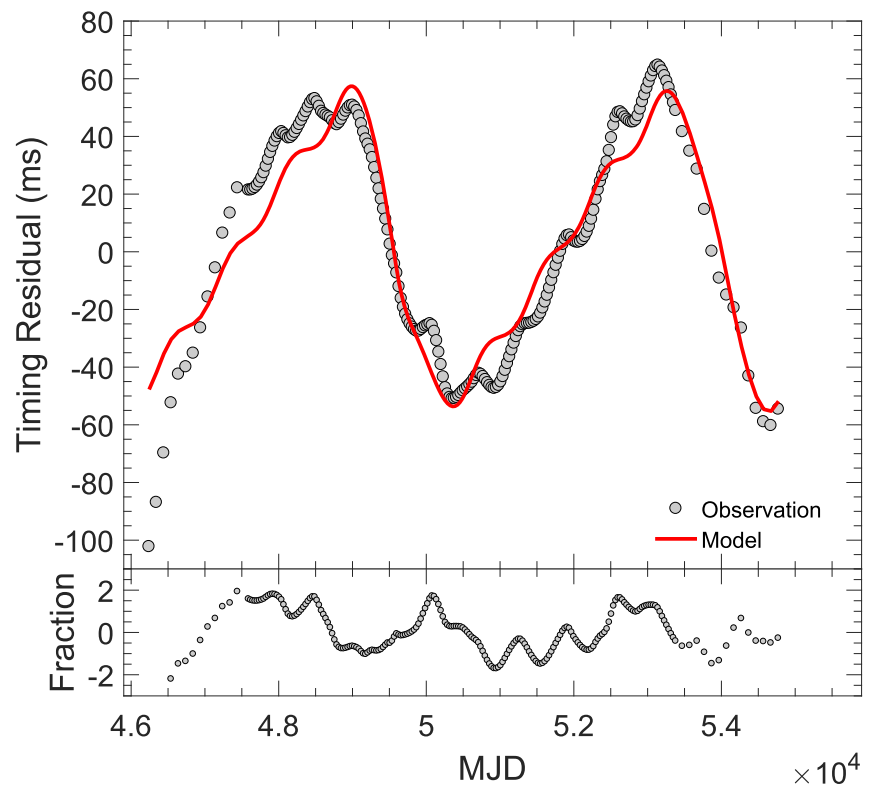

Figure 6. The timing residual for PSR B0919+06. Upper panel: the dotted curve and solid curve correspond to the observed and fitted timing residual of PSR B0919+06, respectively. Lower panel: the time residual after the subtraction of our fitted time noise (red curve in the upper panel) in units of $\sigma_{i}=10 \mathrm{~ms}$.

When the spin velocity of the WD, $\omega_{\mathrm{c}}$, differs from the angular velocity of the orbit $n$, the angle $\phi$ of Equations (10) and (11) is given by $\phi=\left(\omega_{\mathrm{c}}-n\right) t$ instead of a constant angle. Notice that $\phi=\left(\omega_{\mathrm{c}}-n\right) t$ is satisfied only in the case that the equatorial plane of the WD and the pulsar orbit are coplanar. However, in general the misalignment between these two planes (the equatorial plane of the WD and the orbital plane of the pulsar) needs to be considered, as shown in the top right of Figure 2. This will only complicate the expression of $\phi$ by projecting $\omega_{\mathrm{c}} t$ onto the orbital plane, which is done in a practical simulation.

The first term in Equation (11) is equivalent to the tidal potential in the circular orbit case. This term gives rise to a tidal bulge with its axis of symmetry pointing toward the guiding center of the pulsar's orbit. The rest terms of Equation (11) are results of the orbital eccentricity. The second term is the socalled radial tide and the third is the librational tide, in which the former varies with $\cos n t$ and the latter with $\sin n t$, respectively. These three different terms exert tidal force on the WD, which alters its shape and produces perturbations to the orbital motion of the pulsar.

The potential $V_{\mathrm{q}}$ of Equation (11) is actually the tidal potential on the surface of the WD exerted by the pulsar. The WD itself also has potential on the point $P$, which is denoted by

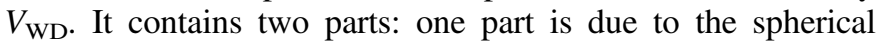
body, and the other noncentral part stems from the matter distribution between the surface of the deformed body and the mean sphere. Apparently the former is independent of the angle $\beta$ of Equation (2) in the main text. In contrast, the latter, denoted by $V_{\mathrm{nc}}$, depends on the angle $\beta$. The gradient on the former and latter potentials produces zero and nonzero forces, respectively. The latter corresponds to the noncentral part of the external potential of the deformed WD at the same point $P$ on the surface of the WD. It can be obtained by the same technique of a planet-satellite system (Murray \& Dermott 1999):

$$
V_{\mathrm{nc}}=-k_{2} G M_{\mathrm{p}} \frac{R_{\mathrm{c}}^{2}}{a^{3}}\left(\frac{R_{\mathrm{c}}^{3}}{a^{3}}\right) P_{2}(\cos \beta),
$$

where $k_{2}$ is the so-called Love number, given by $k_{2}=$ $\left(R_{\mathrm{c}} \epsilon_{2} / \xi\right)-1$ (with $\xi=\frac{m_{\mathrm{p}}}{m_{\mathrm{c}}} \frac{R_{\mathrm{c}}^{4}}{a^{3}}$ ). Substituting such a $k_{2}$ into Equation (12) and comparing with, $V_{\mathrm{c}}^{\prime}$, the first term on the right-hand side of Equation (11), we have

$$
\frac{V_{\mathrm{nc}}}{V_{\mathrm{c}}^{\prime}}=\epsilon_{2} \frac{M_{\mathrm{c}}}{M_{\mathrm{p}}}
$$

With $\epsilon_{2} \sim 10^{-1}$, and by the mass ratio of $M_{\mathrm{c}} / M_{\mathrm{p}} \approx 10^{-1}$ as shown in Tables 1 and 2, the contribution of the $V_{\mathrm{nc}}$ of Equation (12) to the potential at point $P$ is much less than that of the $V_{\mathrm{q}}$ of Equation (11), and is thus ignored.

Now we discuss the amplitude of peaks as shown in Figure 1 (a). The potential of Equation (11) corresponds to a force, determined by the gradient of the pulsar's external potential on the WD. And such a force in turn corresponds to a torque $\Gamma$ on the WD, $\Gamma=\boldsymbol{r} \times \boldsymbol{F}$. Hence, the magnitude of the torque on the WD due to, e.g., the radial tide, as shown in the second term on the right-hand side of Equation (11), is given by $\Gamma=-m_{\mathrm{c}} \frac{\partial V_{\mathrm{r}}}{\partial \beta}$, where

$$
\frac{\partial V_{\mathrm{r}}}{\partial \beta}=-3 e G \cos n t \frac{M_{\mathrm{p}}}{a} \frac{R_{\mathrm{c}}^{2}}{a^{2}} \frac{\partial P_{2}}{\partial \beta}=\frac{9}{2} e G \frac{M_{\mathrm{p}}}{a} \frac{R_{\mathrm{c}}^{2}}{a^{2}} \cos n t \sin 2 \beta .
$$

By using Equation (14), the torque on WD can be obtained:

$$
\Gamma=\frac{9}{2} e G \frac{M_{\mathrm{p}} M_{\mathrm{c}}}{a} \frac{R_{\mathrm{c}}^{2}}{a^{2}} \cos n t \sin 2 \beta .
$$

We have $\Gamma \approx 1 \times 10^{46} \mathrm{~g} \mathrm{~cm}^{2} \mathrm{~s}^{-2}$ with the parameters found in Equation (1) in the main text.

Thus, in the study of short-term effects, the torque originated in the radial tide of Equation (14) varies with $\propto \cos n t \sin 2 \beta$. Comparatively, the torque induced by the librational tide varies with a different function of time, which can be given by the third term on the righthand side of Equation (11). They both contribute to the modulation at the timescale of the orbital period.

In the study of the secular effects induced by tide, the angle $\beta$ in Equation (16) is replaced by the angle of the phase shift in the long term. This angle determines whether the tide bulge is carried ahead of (or lags behind) the tide-raising pulsar, which is related with the energy dissipation over one orbit.

The torque $\Gamma$ given by Equation (15) can vary the spin angular momentum of the WD by

$$
\Gamma=\dot{S}_{\mathrm{c}}=d\left(I_{\mathrm{c}} \omega_{\mathrm{c}}\right) / d t \approx \dot{I}_{\mathrm{c}} \omega_{\mathrm{c}},
$$

where the spin of the WD is assumed to be unchanged. In other words, the torque-induced change of the angular momentum of the WD can be attributed to the change of the moment of WD inertia alone, because the shape change from one state to another at two different times is equivalent to either a change of the moment of inertia with a constant spin velocity or a change of spin velocity with a constant moment of inertia. 
As we study the behavior of the pulsar-WD binary at a timescale of hours, which is a few or tens of its orbital period, the damping of the oscillation amplitude of the hydrostatic equilibrium and the phase lag between the oscillation of the WD and external force are negligible. In such a case, it is a good approximation to treat the change of the moment of inertia as being proportional to the external torque, which is analogous to that of the forced oscillation of a spring system at a timescale that is much shorter than the lifetime of its oscillation.

Hence, at a short timescale, e.g., $\delta t=P_{\mathrm{b}} / 4$, the change of the moment of inertia of the WD in one orbit can be estimated via Equation (16):

$$
\delta I_{\mathrm{c}} \approx \Gamma \delta t / \omega_{\mathrm{c}}
$$

Such a tide-induced variation of $\delta I_{\mathrm{c}}$ reflects a shape change of the WD, which can be compared with the moment of inertia of the WD (with a spin velocity given by Table 1):

$$
q=\frac{\delta I_{\mathrm{c}}}{M_{\mathrm{c}} R_{\mathrm{c}}^{2}} \sim 10^{-1} .
$$

With different timescales in Equation (17), e.g., $\delta t=P_{\mathrm{b}} / 4$ or $\delta t=P_{\mathrm{b}}$, the value of $q$ in Equation (18) can be 0.12 and 0.48 , respectively, which corresponds to an amplitude of a tidal bulge of $\epsilon_{2} \approx(30 \%-70 \%) R_{\mathrm{c}}$.

The perturbing corresponding to the potential of Equation (3) modifies the orbit in a way that is described by the classical celestial dynamics. Consequently, the short-term time delay is affected. The dominant time delay of a pulsar in orbit motion is the Roemer delay, corresponding to a different propagation time to observers as the pulsar moves at different positions of the orbit.

Apparently, if the tidal effect can create additional variability in the orbital elements such as $a, i, U$, and $e$, the Roemer delay of Equation (1) in the main text will vary accordingly.

The effect of the spin-induced quadrupole momentum of the companion star (a main-sequence star) on pulsar timing has been calculated by standard celestial dynamics (Wex 1998), in which the orbital inclination, $\theta$, was defined with respect to the equatorial plane of the companion star. In fact, $\theta$ can be transferred to the usual orbital inclination angle, $i$, defined with respect to the line of sight by

$$
\cos i=\sin \lambda_{\star} \sin \theta \cos \phi+\cos \lambda_{\star} \cos \theta,
$$

where $\lambda_{\star}$ is the angle between the line of sight and the spin of the companion, and $\phi$ is the longitude of the orbital plane in the equatorial coordinate system (Wex 1998). By putting the tideinduced $Q$ into the six orbital elements, the Roemer delay coupling with the tidal effect can be obtained.

More concretely, substituting the potential of the radial tide in Equation (14) and the other two potentials in Equation (11) into $\Gamma=-m_{\mathrm{c}} \frac{\partial V_{\mathrm{r}}}{\partial \beta}$, we actually have a time-varying torque. Furthermore, substituting such a time-varying $\Gamma$ into Equation (16)-Equation (18), and using Equation (2) in the main text, the time-dependent $Q(t)$ can be obtained.

Then by inputting such a varying $Q$ into the perturbation of Equations (47)-(52) of Wex (1998), the perturbation of the six orbital elements can be obtained.
Finally, by substituting the six orbital elements into the Roemer delay of Equation (1) in the main text, $\Delta_{R}$, the time residual originating in an ultra-compact binary with strong tidal effect is obtained. When searching the parameter space of such a theoretical residual by the Monte Carlo method, the best parameter combination is obtained as shown in Table 1. The corresponding timing residual is compared with the observational residual as shown in Figures 1(a) and (b) of the main text. Although the fitting is far from perfect, the main features displayed in the observed time residual are well reproduced in the simulated one.

As shown Figures 1(a) and (b), the horizontal axis corresponds to eight orbital periods ( $868 \mathrm{~s}$ each). The period is a little larger than the fitted orbital period of $855.5 \mathrm{~s}$ as shown in Table 1. This is because the tidal effect is determined by the difference between the orbital frequency and the spin frequency of the WD. As a result, the apparent orbital frequency is lower than that of the true one.

\section{Appendix C X-Ray Observation and Modeling}

On the X-ray band, the area of PSR B0919+06 was observed by XMM-Newton and ROSAT. However, the ROSAT data, with no counts at the position of the pulsar, are not helpful because of the shallow exposures.

XMM Newton observation of PSR B0919+06 was performed on 2007 November 09 (obsID: 0502920101, PI: V. Zavlin) with the European Photon Imaging Camera (EPIC) in imaging mode. We reprocessed the data using Science Analysis Software (SAS) version 14.0.0 and the calibration files updated in 2015 May, following standard steps in the thread page. ${ }^{12}$ More than half of the exposure was strongly contaminated by the high background fluctuation. After screening using the espfilt tool, the resulting effective exposure times were $16.3 \mathrm{ks}, 19.1 \mathrm{ks}$, and $10.4 \mathrm{ks}$, respectively, for MOS1, MOS2, and PN.

We found an X-ray counterpart at the position of PSR B0919 +06 , which is clearly seen in the count images on PN, blurry on MOS2, and barely seen on MOS1 (Figure 7). The standard source detection algorithm emldetect has been applied to the data in the energy range of $0.2-12 \mathrm{keV}$ and as a cross-check in the restricted energy bands $0.2-0.5 \mathrm{keV}, 0.5-1 \mathrm{keV}, 1-2 \mathrm{keV}$, $2-4.5 \mathrm{keV}$, and $4.5-12 \mathrm{keV}$. emldetect detected the X-ray counterpart as a point source on the PN and MOS2 detectors with no detection on MOS1. The best-fit position with a maximum likelihood method was determined at R.A. $=9^{\mathrm{h}} 22^{\mathrm{s}} 14^{\mathrm{s}} .12$, decl. $=6^{\circ} 38^{\prime} 24^{\prime \prime}$. 7 , with an error of $1 !^{\prime \prime} 0$ on PN and at R.A. $=9^{\mathrm{h}} 22^{\mathrm{s}} 14^{\mathrm{s}} 11$, decl. $=6^{\circ} 38^{\prime} 24^{\prime \prime} .5$, with an error of 1 !" 3 on MOS2, which is compatible with the radio coordinates of PSR B0919+06 (at R.A. $=09^{\mathrm{h}} 22^{\mathrm{m}} 14^{\mathrm{s}} .01$, decl. $=+06^{\circ} 38^{\prime} 22^{\prime \prime} 84$, J2000, with the position accuracy of $<1^{\prime \prime}$ (White et al. 1997) and a distance of $2^{\prime \prime}$ between the radio pulsar in the Faint Images of the Radio Sky at Twenty survey and the XMM-Newton best-fit position). Therefore, we consider this X-ray point source to be the counterpart of the PSR B0919 +06 system. Given the low number of counts, a detailed phase study was not allowed. A deeper observation is needed to constrain the emission mechanism.

In the spectral analysis, we extracted from all three detectors the source spectra from a circular region of radius $20^{\prime \prime}$ centered

\footnotetext{
12 http://xmm.esac.esa.int/sas/current/documentation/threads/
} 

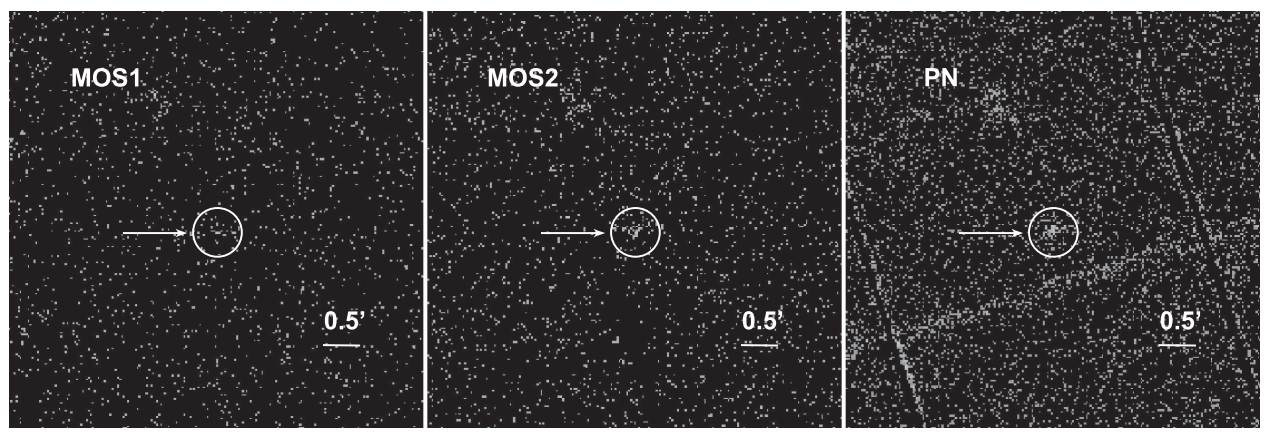

Figure 7. XMM Newton EPIC-MOS1, MOS2, and PN count images of PSR B0919+06 in the energy range of $0.2-10 \mathrm{keV}$. The white circles show the spectral extraction regions with radii of $20^{\prime \prime}$.

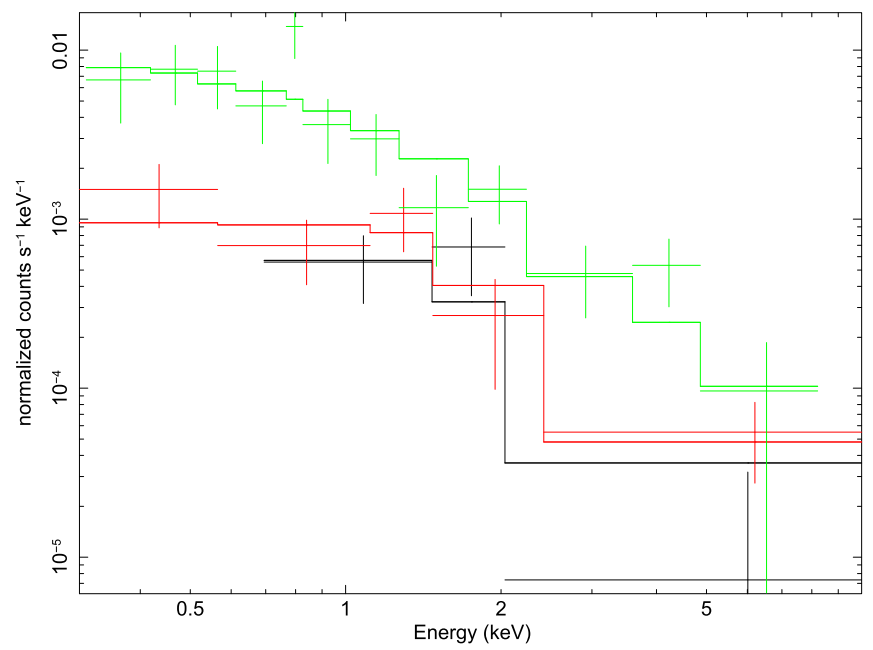

Figure 8. XMM Newton background-subtracted spectra of PSR B0919+06 fitted with an absorbed power-law model. The black crosses are data from MOS1, the red crosses are from MOS2, and the green are from PN. All error bars are reported at the $90 \%$ confidence level. The solid curves are the absorbed power-law model fitted with the same values of $N_{\mathrm{H}}$ and the photon index but different values of norms for MOS1 (black), MOS2 (red), and PN (green), respectively.

on the position of the pulsar and the background counts from nearby source-free regions. The background-subtracted spectra were then grouped with at least nine counts per bin and a maximum oversampling of the spectral energy resolution of a factor of 3 (Figure 8). Using the C-statistic in the XSpec analysis package (v12.8.2), an absorbed ${ }^{13}$ power-law model gave a reasonable fit with a $\mathrm{C}$-statistic of 16.01 over 15 degrees of freedom, the best-fit absorptionof $N_{\mathrm{H}}<7.6 \times 10^{20} \mathrm{~cm}^{-2}$, and the power-law index of $\Gamma=1.73(1.47-2.20)$, while a purely blackbody model cannot provide an acceptable fit. All errors are reported at the $90 \%$ confidence level. The unabsorbed luminosity in $0.3-10 \mathrm{keV}$ was estimated as $2.58 \times 10^{30} \mathrm{erg} \mathrm{s}^{-1}$, $4.1 \times 10^{30} \mathrm{erg} \mathrm{s}^{-1}$, and $4.8 \times 10^{30} \mathrm{erg} \mathrm{s}^{-1}$, respectively, for MOS1, MOS2, and PN, assuming a distance of $1.2 \mathrm{kpc}$.

The light-curve of $0.2-10 \mathrm{keV}$ was extracted from the same regions in the spectra analysis, using standard SAS tools. Photon arrival times were corrected to the solar system barycenter. Since the signals were too weak for MOS1 and

\footnotetext{
${ }^{13}$ We took into account the effects of interstellar absorption along the line of sight through the TBABS model with the vern cross-sections (Verner et al. 1996) and the wilm abundances (Wilms et al. 2000).
}

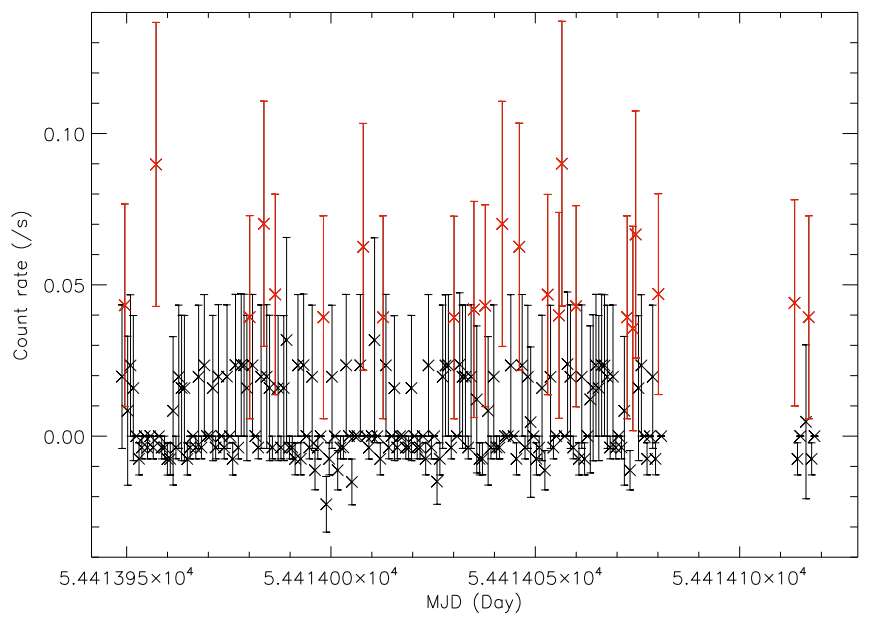

Figure 9. XMM EPIC-PN background-subtracted light curve of PSR B0919 +06 in the $0.2-10 \mathrm{keV}$ band. The red data points are highlighted for having positive values considering error bars.

MOS2, we only show the light curves of EPIC-PN with time bins of 1 minute in the right-hand side of Figure 9 highlighting data points with signal-to-noise ratios larger than $1 \sigma$.

\section{References}

Althaus, L. G., \& Benvenuto, O. G. 1997, ApJ, 477, 313

Barker, B. M., \& O'Connell, R. F. 1975, PhRvD, 12, 329

Brisken, W. F., Fruchter, A. S., Goss, W. M., Herrnstein, R. M., \& Thorsett, S. E. 2003, AJ, 126, 3090

Camilo, F., Lorimer, D. R., Freire, P., Lyne, A. G., \& Manchester, R. N. 2000, ApJ, 535, 975

Chatterjee, S., Cordes, J. M., Lazio, T. J, et al. 2001, ApJ, 550, 287

Cui, W. 1997, ApJL, 482, L163

Di Salvo, T., Burderi, L., Riggio, A., Papitto, A., \& Menna, M. T. 2008, MNRAS, 389, 1851

Frank, J., King, A., \& Raine, D. 2002, Accretion Power in Astrophysics (Cambridge: Cambridge Univ. Press)

Gong, B. P., \& Li, Y. P. 2013, arXiv:1302.6299

Han, J., et al. 2016, MNRAS, 456, 3413

Hartman, J. M., Patruno, A., Chakrabarty, D., et al. 2008, ApJ, 675, 1468

Hobbs, Lyne, \& Kramer 2004, MNRAS, 353, 1311

Hobbs, G., Lyne, A. G., \& Kramer, M. 2010, MNRAS, 402, 1027

Illarionov, A. F., \& Sunyaev, R. A. 1975, A\&A, 39, 185

Johnston, H. M., \& Kulkarni, S. R. 1991, ApJ, 368, 504

Lamb, F. K., Pethick, C. J., \& Pines, D. 1973, ApJ, 184, 271L

Lyne, A. G., Hobbs, G., Kramer, M., Stairs, I., \& Stappers, B. 2010, Sci, 329,408

Marsh, T. R., \& Nelemens, G. 2004, MNRAS, 350, 113

Murray, C. D., \& Dermott, S. F. 1999, Solar System Dynamics (Cambridge: Cambridge Univ. Press) 
Perera, B. B. P., Stappers, B. W., Weltevrede, P., Lyne, A. G., \& Bassa, C. G. 2015, MNRAS, 446, 1380

Rankin, J. M., Rodriguez, C., \& Wright, G. A. E. 2006, MNRAS, 370, 673

Ransom, S. M., Cordes, J. M., \& Eikenberry, S. S. 2003, ApJ, 589, 911

Rosen, S. R., Webb, N. A., Watson., M. G., et al. 2016, A\&A, 590, A1

Shabanova, T. V. 2010, ApJ, 721, 251

Shabanova, T. V., Pugachev, V. D., \& Lapaev, K. A. 2013, ApJ, 775, 2

Shibata, S., Watanabe, E., Yatsu, Y., Enoto, T., \& Bamba, A. 2016, ApJ, 833,59

Verbunt, F., \& Rappaport, S. 1998, ApJ, 332, 193
Verner, D. A., Ferland, G. J., Korista, K. T., \& Yakovlev, D. G. 1996, ApJ, 465,487

Wahl, H. M., Orfeo, D. J., Rankin, J. M., \& Weisberg, J. M. 2016, MNRAS, 461,3740

Wex, N. 1998, MNRAS, 298, 67

White, R. L., Becker, R. H., Helfand, D. J., \& Gregg, M. D. 1997, ApJ, 475,479

Wilms, J., Allen, A., \& McCray, R. 2000, ApJ, 542, 914

Yao, J. M., Manchester, R. N., \& Wang, N. 2017, ApJ, 835, 29

Zhang, S. N., Yu, W., \& Zhang, W. 1998, ApJ, 494, 71 